CAHIERS DE

NARRATOLOGIE

\section{Cahiers de Narratologie}

Analyse et théorie narratives

$14 \mid 2008$

Prose d'idées : formes et savoirs

\title{
Roman picaresque et prose d'idées : Guzmán de Alfarache de Mateo Alemán
}

Jean-Louis Brau

\section{(2) OpenEdition}

Journals

Édition électronique

URL : http://journals.openedition.org/narratologie/534

DOI : $10.4000 /$ narratologie.534

ISSN : 1765-307X

Éditeur

LIRCES

Référence électronique

Jean-Louis Brau, «Roman picaresque et prose d'idées: Guzmán de Alfarache de Mateo Alemán », Cahiers de Narratologie [En ligne], 14 | 2008, mis en ligne le 05 janvier 2011, consulté le 19 avril 2019 URL : http://journals.openedition.org/narratologie/534; DOI : 10.4000/narratologie.534

Ce document a été généré automatiquement le 19 avril 2019

\section{(c) $($ i) $(9)$}

Cahiers de Narratologie - Analyse et théorie narratives est mis à disposition selon les termes de la licence Creative Commons Attribution - Pas d'Utilisation Commerciale - Pas de Modification 4.0 International. 


\title{
Roman picaresque et prose d'idées : Guzmán de Alfarache de Mateo Alemán
}

\author{
Jean-Louis Brau
}

1 En octobre 2006, lors de la journée sur la prose d'idées organisée par le CIRCLES, MarieLaure Acquier étudiait les "Enjeux et ressorts de la prose d'idées en Espagne au XVII siècle" en illustrant son propos avec une analyse de l'œuvre d'Antonio López de Vega ${ }^{1}$ dans laquelle elle se posait la question de l'opérativité de la notion de prose d'idées à l'époque baroque. Je renvoie à son excellente synthèse et je retiendrai surtout deux de ses remarques. La première, c'est que de nombreux auteurs de l'époque, qualifiés de "polygraphes" et dont Quevedo est l'exemple le plus illustre (il a écrit des traités de politique, des romans, des satires, des poésies), de nombreux polygraphes de l'époque donc ont abordé dans leurs écrits plusieurs genres ressortissant soit à la prose d'idées, soit à la littérature, dans la mesure où l'on peut opposer ces deux catégories d'une manière claire et tranchée et c'est là tout le problème. Marie-Laure Acquier a justement bien montré combien il est difficile de donner une définition précise de la prose d'idées et combien les frontières entre prose d'idées et littérature sont souvent floues (je renvoie à son analyse). C'est ce qui la conduit à "emprunter à Stéphane Pujol son expression Littérature d'idées qu'il donne pour équivalente de celle de prose d'idées."

2 C'est la deuxième remarque que je retiendrai car elle me semble intéressante dans la perspective de l'analyse du roman picaresque qui me préoccupe. C'est pourquoi, avant d'entrer dans le vif du sujet, je reviendrai sur cette idée d'opposition ou d'équivalence entre prose d'idées et littérature d'idées qu'il me paraît important de creuser un peu avant d'aborder le roman picaresque.

3 De tout temps, la prose d'idées a utilisé, dans un souci didactique, toutes sortes d'exemples ou d'exempla, pour utiliser le terme latin consacré par la critique, soit pour illustrer de manière simple et compréhensible par tous des raisonnements compliqués, soit, et ce n'est pas exclusif, pour reposer et divertir le lecteur ou l'auditeur, fatigué par une longue concentration sur des sujets arides et rébarbatifs. D'où l'utilisation de nombre 
de procédés qui vont du dialogue (on pense à Platon et à ses innombrables épigones) à la nouvelle intercalée, en passant par une infinité d'exempla de toutes sortes et de toutes origines. Au Moyen-Age, le genre oratoire puise abondamment dans les répertoires d'exempla qui circulent en Europe comme la célèbre Disciplina clericalis de Pedro Alfonso, recueil de contes et de sentences d'origines diverses qui date du $\mathrm{XI}^{\mathrm{e}}$ siècle. ${ }^{3}$ Ces exempla émaillent les sermons des prédicateurs chargés de faire comprendre à un peuple frustre et ignorant les difficiles concepts du dogme religieux. Mais, bien entendu, c'est toujours le discours idéologique qui prime et les exempla n'apparaissent que ponctuellement comme de simples illustrations.

4 Inversement, c'est un truisme de dire que la littérature contient toujours une dimension idéologique plus ou moins importante et plus ou moins explicite. Cette dimension est explicite lorsque le narrateur ou l'un des personnages exprime directement ses idées sur un sujet. Dans le domaine hispanique, un exemple extrême apparait dans une œuvre du XIII siècle, El conde Lucanor (Le comte Lucanor) de l'infant Don Juan Manuel, qui se présente comme un dialogue entre le comte Lucanor et son valet-confident Patronio au sujet du mariage $e^{4}$. Patronio conte à son maître des anecdotes qu'il termine par une morale très courte qui découle de l'action. C'est le principe même de la fable. D'autre fois, la plupart du temps d'ailleurs, la dimension idéologique est implicite et c'est le lecteur, s'il est un tant soit peu lectant, pour employer le terme forgé par Vincent Jouve, c'est-à-dire s'il est doté d'une certaine capacité réflexive, qui doit la déduire des personnages et de leurs actions $^{5}$.

5 Il y a donc d'importantes affinités entre la prose d'idées et la littérature d'idées qui combinent toutes deux idéologie et fiction. Ce qui faisait dire à Marie-Laure Acquier « Ni dans l'une ni dans l'autre, la fiction ne fait figure d'élément discriminant $»^{6}$. Je serai peutêtre moins catégorique et je nuancerai cette affirmation car je pense que si la présence de la fiction n'est pas génériquement discriminante, ce qui l'est c'est son importance, notamment quantitative, par rapport à l'idéologie. Autrement dit, je crois que la distinction entre prose d'idées et littérature d'idées est une affaire de dosage entre le discours idéologique et les exempla, selon l'importance relative que l'auteur accorde à l'un ou à l'autre élément dans son texte. Toutes deux (prose d'idées et littérature d'idées) observent le précepte horacien du mixit utile dulci (mêler l'utile à l'agréable ${ }^{7}$ en variant les proportions respectives des deux concepts selon que l'accent est mis sur le message idéologique ou sur la fiction chargée de captiver et de divertir le lecteur.

6 Or, il n'est pas toujours facile de trancher entre la prose d'idées et la littérature d'idées car certaines oeuvres se situent à la frontière entre les deux et nécessitent une analyse poussée qui d'ailleurs ne donne pas toujours des résultats indiscutables si l'on en juge par les commentaires souvent très divergents des critiques sur ces oeuvres complexes. C'est le cas du roman picaresque, intéressant à plus d'un titre, d'abord parce qu'il ouvre le chemin qui conduira au roman moderne, ensuite, et c'est le sujet de mon étude, parce qu'il a été l'objet de commentaires très variés, parfois contradictoires, selon la proximité plus ou moins grande des oeuvres qui en font partie avec la prose d'idées.

7 Je commencerai cette analyse par un bref historique, qui me parait indispensable car il me semble que le contexte historique et littéraire doit être présent à l'esprit si l'on veut bien comprendre la nature du roman picaresque et ses relations avec la prose d'idées. J'analyserai ensuite les principaux romans qui constituent le genre et parmi lesquels je m'attarderai plus particulièrement sur celui qui est considéré comme l'achétype du 
roman picaresque, le Guzmán de Alfarache de Mateo Alemán qui présente d'importantes affinités avec la prose d'idées.

8 Le roman picaresque européen naît en Espagne vers le milieu du XVI siècle avec la publication en 1554 d'un petit livre comique anonyme intitulé La vida de Lazarillo de Tormes $y$ de sus fortunas $y$ adversidades ${ }^{8}$. Pourquoi l'Espagne et pourquoi à ce moment-là ? Pour répondre le plus brièvement possible à cette question, je rapellerai que de nombreuses causes historiques, économiques, sociales et culturelles se sont combinées pour faire éclore ce genre littéraire, nouveau dans la littérature européenne et qui aura un succès inouï auprès du public.

9 Disons pour résumer (je renvoie à la magnifique étude de Maurice Molho sur le roman picaresque espagnol, dans la bibliothèque de La Péïade) ${ }^{9}$ que l'Espagne du XVI ${ }^{e}$ siècle, si elle connait comme le reste de l'Europe la prolifération des gueux de toutes sortes (mendiants, voleurs, truands, etc.), à la suite des guerres continuelles, des pestes et des famines qui ravagent le pays, elle s'en distingue sur un point. En effet, socialement, l'Espagne du Siècle d'Or est divisée en deux grande castes, la caste supérieure représentée par la noblesse grande et petite et la caste inférieure qui regroupe tous les roturiers, artisans, commerçants, paysans et gueux. Ceci n'est pas particulier à l'Espagne. Ce qui l'est en revanche c'est ce qui sépare irrémédiablement ces deux castes, le concept de l'honneur basé sur le lignage, c'est-à-dire sur la naissance, qui, selon Maurice Molho " devient en Espagne le principe recteur de toute vie sociale et morale $»^{10}$

10 Là-dessus, se greffe le problème historique de la coexistence en Espagne de trois grandes communautés ethniques ayant chacune sa religion (chrétiens, juifs et musulmans). A la fin du XVe siècle, les Rois Catholiques mettent fin à la Reconquête et unifient l'Espagne. Ils créent alors le Tribunal de la Sainte-Inquisition afin de pourchasser les hérétiques. Le concept de sang pur va alors s'imposer et diviser la société espagnole en deux castes, celle des "vieux chrétiens", fiers de la pureté de leur sang et qui jouissent de tous les privilèges, et celle des «nouveaux chrétiens » (juifs et maures convertis), méprisés et exclus des emplois intéressants réservés aux vieux chrétiens. Les nobles, qui font partie de la caste dominante, et qui sont exonérés des impôts auxquels sont assujettis les roturiers, affichent orgueilleusement leur supériorité due à leur naissance qui garantit un sang pur et qui rappelle les services rendus aux rois par de nombreuses générations. C'est pourquoi ils s'estiment seuls détenteurs de l'honneur qui leur impose un comportement digne et un mépris souverain des activités productives réservées à la classe inférieure des roturiers. A cet honneur, fondé sur la naissance illustre, le héros picaresque opposera un anti-honneur fondé sur la naissance ignominieuse.

11 D'autre part, pour comprendre l'apparition et le sens du roman picaresque, il faut tenir compte du contexte littéraire de l'époque. Avant son apparition, deux grands genres dominent la littérature espagnole en prose : les livres de chevalerie et les pastorales, deux types de récits idéalistes. Le premier exalte les sublimes vertus guerrières, morales et amoureuses du parfait chevalier, défenseur des opprimés, modèle de courage et de fidélité, car il est de haute naissance, souvent fils de roi ou de prince, et dont l'archétype est le célébrissime Amadis de Gaule, sans doute composé au XIV siècle, mais qui fut redécouvert au XVI ${ }^{\mathrm{e}}$ et qui eut pendant tout le Siècle d'Or un très grand succès et une très nombreuse descendance. L'autre genre (la pastorale, dont l'archétype est La Diana de Montemayor) décrit la vie bucolique et amoureuse des innocents bergers, poètes et musiciens en contact étroit avec une nature idyllique. Ces récits idéalistes permettaient 
au lecteur ou à l'auditeur ${ }^{11}$ de s'évader pendant un moment de la morne réalité quotidienne.

12 Le Lazarillo de Tormes va prendre le contrepied de ces utopies merveilleuses en relatant les mésaventures, la plupart du temps comiques, d'un miséreux, né dans la fange, fils d'un voleur minable et d'une femme de rien, serviteur de maitres ignobles ou lamentables qui lui donnent des leçons d'hypocrisie, d'immoralité et de cynisme. Dans le panorama littéraire idéaliste de l'époque, ce petit livre détonne par son originalité profonde qui tient au choix d'un antihéros, à la forme qui est celle de la confession faite à la première personne, à la tonalité humoristique prononcée du récit et à l'autodérision permanente.

13 On a voulu voir dans ce court roman ${ }^{12}$ un brûlot anticlérical et antinobiliaire (écrit d'ailleurs peut-être par un ecclésiastique réformateur) car des sept maîtres que Lazarillo sert successivement, cinq sont des prêtres peu reluisants et un autre un hidalgo falot et pitoyable, victime de sa religion de l'honneur. Certains commentateurs font aussi état, pour conforter cette thèse, de quelques rares apartés sarcastiques du protagoniste qui commente brièvement, avec une apparente naïveté, le comportement scandaleux ou pathétique de ses différents maîtres. Pour ma part, je me rangerai à l'avis d'un grand hispaniste, fin connaisseur de la littérature espagnole du Siècle d'Or, Marcel Bataillon, qui n'y voit qu'un livre facétieux («un libro de burlas») qui reprend l'anticléricalisme folklorique des fabliaux du Moyen-Age et qui ne fait qu'une critique très indirecte et très peu appuyée de l'honneur mondain des hidalgos. Nulle trace de prose d'idées dans ce petit bijou littéraire, même si l'auteur anonyme suggère dans son subtil prologue qu'il y a matière à réflexion pour le lecteur désireux d'aller au fond des choses.

14 Le Lazarillo de Tormes eut beaucoup de succès, mais pas de descendance immédiate. Il fallut attendre presque un demi-siècle avant la parution d'un roman qui reprenait la formule fictionnelle du génial précurseur en l'amplifiant considérablement à tous les niveaux. En effet, en 1599, un obscur fonctionnaire, Mateo Alemán, quelque peu polygraphe lui aussi ${ }^{13}$, publie en 1599 la Première partie de Guzmán d'Alfarache, guette-chemin de la vie humaine ${ }^{14}$ qui se présente, de même que le Lazarillo de Tormes, comme l'autobiographie d'un gueux qui vit de nombreuses aventures tragicomiques au service d'un certain nombre de maîtres. Mais si le Lazarillo de Tormes et le Guzmán de Alfarache ont en commun ce mince canevas fictionnel, on peut dire que tout les sépare.

15 Au niveau de la fiction tout d'abord, le roman d'Alemán est beaucoup plus long et beaucoup plus complexe que son bref prédécesseur. Le héros, Guzmán, parcourt un espace bien plus étendu que celui du petit Lazarillo dont l'histoire nous mène en quelques semaines de Salamanque à Tolède. Guzmán, parti enfant de Séville, vit quelques années à Madrid, rejoint Rome via Barcelone et la Méditerranée. Après de nombreuses années passées dans la ville sainte comme mendiant puis au service de grands personnages, un cardinal et un ambassadeur de France, il revient en Espagne où, homme déjà mûr, il vit de nouveau quelques années à Madrid comme homme d'affaires véreux, puis comme étudiant en théologie et enfin comme proxénète de sa propre épouse avant de regagner Séville et de finir comme forçat aux galères. Cette longue histoire est peuplée d'une multitude de personnages secondaires rencontrés par le héros au cours de son périple, ce qui génére un très grand nombre de péripéties de toutes sortes. Mais ce n'est pas tout, car le récit foisonnant de l'histoire du gueux est interrompu sept fois par l'insertion de nouvelles d'une assez grande extension intercalées dans la trame narrative principale. Au niveau fictionnel, il n'y a donc aucune commune mesure entre le court récit facétieux du Lazarillo de Tormes qui relate six ou sept anecdotes comiques et le Guzmán de Alfarache 
,véritable roman-fleuve qui retrace toute une vie longue et mouvementée. On observe donc déjà, au niveau fictionnel, une opposition très forte entre l'esthétique de la Renaissance, représentée ici par le Lazarillo de Tormes et caractérisée par la sobriété et la simplicité, et l'esthétique du Baroque, celle du Guzmán de Alfarache, avec sa profusion ornementale, son goût de la surcharge. Mais ce n'est pas là l'essentiel, aussi, je ne m'y attarderai pas.

16 Ce qui distingue surtout le Guzmán du Lazarillo, c'est sa structure générale, extrêmement digressive. En effet, le récit de la vie du protagoniste est à chaque instant entrecoupé de digressions très variées et souvent très longues (certaines occupent plusieurs chapitres), parmi lesquelles prédominent les sermons de type religieux ou plus généralement moralisateurs. Le récit se développe ainsi sur deux plans, le plan fictionnel des péripéties qui est celui du récit autobiographique des aventures picaresques tragicomiques $d u$ protagoniste Guzmán («las consejas » dans le vocabulaire d'Alemán), et le plan de la "leçon» qui est celui des innombrables considérations morales explicitement développées par le narrateur (ce que l'auteur appelle «los consejos»). Et ces deux plans sont inextricablement mêlés car chaque expérience du gueux suscite en lui des réflexions, le plus souvent amères et désabusées sur l'homme et sur la société.

17 Mais il y a plus, car sur cette structure principale bipolaire (consejas/consejos) vient se greffer une structure secondaire. En effet, les digressions constantes, qui interrompent à tout propos le récit picaresque, ne sont pas homogènes car la partie doctrinale des "sermons » est elle-même truffée de cellules narratives plus ou moins développées qui s'intercalent dans ces sermons et qui vont du jeu de mots ou de l'anecdote amusante jusqu'à la nouvelle de style italien ou mauresque en passant par la fable, le conte folklorique, l'apologue mythologique, l'exemple tiré de l'Antiquité ou de la Bible ou la légende hagiographique. Ces innombrables exempla illustrent les sermons en atténuant leur aridité et en donnant plus de vie et d'allure facétieuse au récit, mais aussi en contribuant fortement à compliquer la structure principale déjà complexe.

18 Cette accumulation d'exempla d'une variété extrême, mais dont la redondance sémantique atteint parfois la limite du supportable pour le lecteur, répond à une double préoccupation. D'une part, elle traduit un souci d'efficacité maximale (le narrateur dit à ce sujet: "Mais, comme le but que je me suis fixé est celui de fabriquer un homme parfait, chaque fois que je trouve des pierres pour l'édifice, je les entasse. $\aleph^{15}$, d'autre part elle répond à une préoccupation esthétique à laquelle j'ai fait allusion plus haut et qui est le goût typiquement baroque pour l'ornementation luxuriante qu'on retrouve à l'époque, notamment dans les arts plastiques, la peinture, la sculpture, dans la musique aussi.

19 Au passage, on peut apprécier tout au long du livre la culture et l'érudition de Mateo Alemán qui avait fait des études de médecine et qui était sans doute, comme son contemporain Cervantès, un lecteur vorace, grand connaisseur de la littérature de son temps, aussi bien profane que sacrée, de la Bible particulièrement, mais aussi de la prose d'idées et des miscellanées (mélanges) à la mode au Siècle d'Or, ces fourre-tout qui traitaient toutes sortes de sujets propres à satisfaire la curiosité des lecteurs comme la Silva de varia lección (Forêt de sujets divers) de Pero Mejía (Séville 1540), l'un des livres les plus lus en Europe, ou le Jardín de flores curiosas (Jardin de fleurs curieuses) de Antonio de Torquemada (Salamanque, 1570) lui aussi largement diffusé en Europe. Toutes ces lectures ont nourri la pensée d'Alemán et lui ont fourni la multitude d'exempla qui émaillent son roman comme il l'avoue honnêtement dans son prologue "Au lecteur avisé » : « Tout n'est pas de moi ; j'ai beaucoup emprunté à des hommes doctes et saints » 
${ }^{16}$. Car ce qui comptait pour les écrivains de l'époque, ce n'était pas l'originalité à tout prix, mais le souci de montrer une bonne érudition et de s'appuyer sur l'autorité d'auteurs prestigieux.

20 Mais n'oublions pas que le narrateur fictif, c'est le gueux qui raconte sa vie et qui prend donc à son compte la culture et l'érudition de l'auteur, ce qui aurait pu paraître invraisemblable. C'est pourquoi, soucieux de la vraisemblance d'un récit dans lequel un misérable gueux est capable de faire des sermons dignes du meilleur prédicateur, Alemán prend bien soin de faire de son protagoniste un homme intelligent et instruit. Dans sa préface, intitulée "Déclaration pour la compréhension de ce livre", il présente son personnage en ces termes : «On présuppose que Guzmán d'Alfarache, notre gueux, ayant été fort bon étudiant, ayant appris le latin, la réthorique et le grec..., étant ensuite revenu d'Italie en Espagne, poursuivit ses études, avec l'intention d'entrer dans les ordres ; mais il les abandonna pour retomber dans ses vices.Et ce n'est pas invraisemblable ni hors de propos si dans cette première partie il lui arrive d'écrire quelques passages de doctrine ; car il semble au contraire très plausible d'en attribuer l'usage à un homme de bon jugement, cultivé et rendu sage par le temps, et qui met à profit les loisirs qu'il a sur la galère $»^{17}$

21 Pour revenir à la luxuriance extrême du récit, elle rend difficile, et, il faut bien le dire, souvent ennuyeuse, la lecture de l'oeuvre, surtout pour un lecteur moderne. Déjà, au $\mathrm{XVIII}^{\mathrm{e}}$ siècle, trouvant indigeste la lecture du livre, Lesage, qui appréciait le récit des aventures picaresques du gueux, publie en 1732 l'«Histoire de Guzmán d'Alfarache,

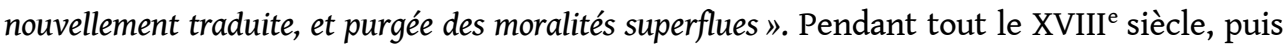
au XIX ${ }^{\mathrm{e}}$ et même au XX $\mathrm{X}^{\mathrm{e}}$ siècle, $\mathrm{y}$ compris en Espagne, les commentateurs critiqueront de la même façon l'énorme accumulation de sermons et de moralités qui, selon eux, alourdissent considérablement le récit. Le grand Unamuno lui-même a exprimé à ce sujet un jugement très sévère et très excessif : «Je suis surpris qu'il y ait des gens qui aient pu aller jusqu'au bout d'une telle kyrielle de sermons ennuyeux et vulgaires remplis de la philosophie la plus grossière ${ }^{1 .}{ }^{18}$ Tous ces critiques n'ont pas compris, ou n'ont pas voulu tenir compte de l'intention didactique et moralisatrice clairement affirmée par l'auteur, et du fait que la fiction picaresque elle-même obéit, comme on le verra, à cette intention.

22 Pourtant, Alemán, prévoyant une telle réaction, a multiplié les mises en garde au lecteur. Il place en tête de son livre deux prologues. Dans le premier, il s'adresse "Au vulgaire " («Al vulgo ») qui ne s'intéresse qu'aux péripéties et il lui dit: «Tu ne vois pas et tu ne remarques pas les nobles leçons des esprits les plus sublimes et tu te contentes seulement de ce qu'a dit le chien et de ce qu'a répondu le renard. $»^{19}$. Et dans le deuxième prologue, il s'adresse «Au lecteur avisé » ( « ... al discreto lector ») en ces termes : « Ne ris point des péripéties en laissant passer les leçons... ne les jette pas comme des balayures dans la poubelle de l'oubli, recueille, rassemble cette terre,... et je t'assure que tu y trouveras un peu d'or qui t'enrichira. $»^{20}$

23 Il est d'ailleurs le premier à avoir conscience de la lourdeur de certains développements qui peuvent indisposer le lecteur, mais il s'en excuse en évoquant sa préoccupation essentielle qui est de critiquer les vices humains. Ainsi, le narrateur, le gueux donc, qui vient de méditer longuement sur l'une de ses aventures dit: «J'ai fait une digression longue et ennuyeuse, je le vois bien; mais ne t'en étonne point, car le sujet était important. ${ }^{21} \mathrm{Ou}$ encore : «J'entends déjà celui qui est en train de me lire dire qu'il a envie de me jeter dans un coin, parce qu'il est fatigué de m'entendre. Il a mille fois raison; car ce sont des vérités ce dont je traite, c'est pourquoi elles ne sont pas faites pour 
divertir, mais pour faire réfléchir ; non pas pour plaisanter, mais pour être examinées avec le plus grand soin et pour qu'on leur trouve un remède. $»^{22}$ Alemán a donc une conscience très nette de la difficulté de faire passer l'enseignement moral. D'où la nécessité de rendre le récit divertissant pas tous les moyens, vieux principe hérité d'Aristote et surtout d'Horace (c'est le fameux mixit utile dulci que je rappelais plus haut) et qu'Alemán reprend à travers une métaphore en accord avec la tonalité picaresque du récit quand il dit au lecteur: "Pour que tu ne sois pas dégoûté de la purge et que tu ne refuses pas de la prendre à cause de sa mauvaise odeur et de son mauvais goût, mélangeons-la avec un peu d'or, recouvrons-la de quelque chose qui ait bonne apparence $»^{23}$.

24 Pour « dorer la pilule ", il utilise toutes les ressources qui lui permettent d'édulcorer le texte, de divertir le lecteur au moyen de toutes sortes d'exemples amusants, surtout dans la fiction principale, l'autobiographie du gueux. On peut donc presque dire, paradoxalement, que, du point de vue de l'intention, ce sont les aventures picaresques qui représentent, à la manière d'un vaste exemplum, une digression dans le traité de morale constitué par les sermons et les développements d'ordre éthique.

25 Ceci pour la structure de l'oeuvre. En ce qui concerne la thématique des digressions idéologiques, elle est variée, elle aussi, mais on peut y distinguer des thèmes secondaires de satire sociale et/ou morale et des thèmes majeurs dominés par la doctrine religieuse.

26 Pour la satire sociale, on trouve dans le Guzmán une critique sévère des faux mendiants qui pululaient, surtout ceux de Rome organisés en confrérie aux règles strictes, et qui abusaient de la générosité des gens honnêtes en détournant les aumônes destinées aux vrais pauvres. C'était là un problème social grave posé par la mendicité en Espagne aussi à l'époque d'Alemán (de nombreux traités consacrés à ce véritable fléau et aux moyens de le réduire sont publiés et on sait qu'Alemán a entretenu une correspondance avec son ami Cristóbal Pérez de Herrera, protomédecin des galères d'Espagne, qui avait écrit sur la question). ${ }^{24}$ Ce problème préoccupait beaucoup les autorités espagnoles de l'époque. Les Cortes (l'équivalent du parlement) prenaient régulièrement des mesures pour essayer de trouver une solution. L'auteur anonyme du Lazarillo y faisait déjà une fugitive allusion lorsque le petit Lazare rencontre dans une rue de Tolède une procession de mendiants étrangers à la ville que l'on menait par les rues en les fouettant. On en trouve un écho important dans le Guzmán dans lequel de nombreux chapitres sont consacrés à ce problème.

27 Viennent ensuite certains corps de métiers : ceux des domestiques, des cuisiniers et des aubergistes, tous voleurs et hypocrites, des maîtres durs, avares et vaniteux, des apothicaires et des médecins ignorants et cupides, des hommes d'affaires, des financiers et des marchands malhonnêtes. Les institutions ne sont pas épargnées: la milice qui donne lieu à toute sorte de trafics, la justice surtout avec ses juges, ses avocats, ses procureurs, ses greffiers et ses alguazils, tous cupides et malhonnêtes. C'est une satire très large, acerbe, mordante, souvent très violente, qui traduit une vision amère, pessimiste de la société, mais qui ne remet pas en cause ses structures profondes, et surtout ses deux piliers que sont l'église et la monarchie. En ce sens, elle est plus morale que sociale et elle est inspirée par une doctrine religieuse qui est celle de l'église catholique.

28 Cette satire morale traite de différents thèmes secondaires et de deux thèmes majeurs. Parmi les thèmes secondaires, on retrouve, outre le problème de la mendicité évoqué plus haut, les lieux communs de la prose d'idées de l'époque qui proviennent souvent 
d'Aristote à travers Erasme comme l'éloge de l'humilité, de la tempérance, de l'amitié, de l'honnêteté ; la critique sévère de la femme chargée de tous les défauts, (mais plutôt que de mysoginie de la part d'Alemán, il s'agit d'une vision traditionnelle et parfois folklorique de la femme, et d'ailleurs la critique des hommes n'est pas moins sévère); l'amour aussi est condamné mais c'est là encore une critique traditionnelle sans doute assombrie par le pessimisme de l'auteur. Enfin il y a l'argent, thème récurrent, car les difficultés financières du protagoniste sont le plus souvent à la source de ses mésaventures. Le séjour de Guzmán à Madrid, à son retour d'Italie, donne lieu à une critique virulente des milieux d'affaires et de tous les trafics qui s'y faisaient.

Quant aux thèmes majeurs, il y en a deux : ceux de l'honneur et de l'universalité du Mal. L'honneur, si important dans l'Espagne du Siècle d'Or, concept collectif basé sur la naissance et l'argent, est omniprésent dans le Guzmán. Le gueux oppose à cet honneur mondain, source de misère psychologique et de malheur, la philosophie allègre de la vie picaresque faite de renoncement et de soif de liberté (écho du Beatus ille d'Horace). Le narrateur, qui est ce même gueux, mais vieilli et repenti après de très dures épreuves et qui écrit son autobiographie sur les bancs d'une galère, renchérit sur le jeune gueux qu'il a été en affirmant que l'honneur mène les âmes à leur perte, et il oppose au critère collectif de l'honneur mondain fondé sur les apparences, le critère individuel de l'honneur vrai fondé sur la pratique de la vertu et notamment sur l'exercice de la charité. Mais on peut dire qu'Alemán dépasse la satire sociale pour accéder au plan spirituel, car, au-delà de la société, ce qui l'intéresse c'est la nature humaine. C'est le deuxième thème majeur, le plus important, qui apparaît aussi bien dans la fiction picaresque que dans les multiples digressions morales en forme de sermons, celui de l'universalité du Mal.

30 Dans la fiction, l'expérience vitale de Guzmán, qui est né d'un adultère commis entre un renégat fourbe et une ancienne prostituée mariée à un barbon, n'est qu'un long processus de désillusion. Le gueux se meut dans un univers où tout, des hommes aux animaux et mêmes aux choses inertes, n'est que tromperie. Mis à part quelques rares écclésiastiques pétris de bonté, Guzmán n'a rencontré tout au long de sa vie que des égoïstes, des voleurs, des ingrats, des hypocrites. Lui-même est tour à tour trompé et trompeur. Pour lui, la vie est une jungle où, dit-il, "Nous vivons tous en nous tendant des pièges les uns aux autres. ». ${ }^{25}$ Même les nouvelles sentimentales intercalées dans la fiction principale n'échappent pas à cette «maladie contagieuse » qu'est le mensonge. Devant la tromperie générale, le gueux conclut que tous les hommes sont fondamentalement mauvais.

31 Ce pessimisme profond a sans doute en partie sa source dans l'expérience vitale de l'auteur qui appartenait à la classe des conversos, descendants de juifs convertis, méprisés et humiliés par les vieux chrétiens, et souvent persécutés par l'Inquisition. Le Guzmán de Alfarache est rempli de cette amertume et du sentiment de profonde injustice des nouveaux chrétiens dont beaucoup étaient animés d'une foi catholique sincère et qui souffraient terriblement de la méfiance et de l'ostracisme dont ils étaient l'objet dans la société espagnole du Siècle d'Or. Mateo Alemán était de ceux-là et on peut dire que toute la philosophie qui se dégage de la fiction picaresque et des sermons a sa source dans la doctrine religieuse longuement exposée et appliquée rigoureusement. Et dans cette doctrine, un thème se détache, c'est celui du péché originel, fondamental, pierre angulaire de tout l'édifice didactique et narratif. Alemán y revient souvent, c'est pour lui une véritable obsession qui explique le pessimisme profond et la tonalité amère de l'oeuvre entière. Pour le chrétien convaincu qu'il est, le péché originel a irrémédiablement perverti la nature humaine. Il y a là un déterminisme implacable qui 
régit l'humanité entière. Alemán n'y voit aucun remède, du moins terrestre : «Ainsi va le monde, et ce n'est point d'aujourd'hui, car il y a bien longtemps que le pois chiche a une pointe. Point de recours ni de remède : Tel nous l'avons trouvé, et tel nous le laisserons. Il ne faut pas espérer des temps meilleurs, ni croire que fut meilleur le passé. Tout a été, est et sera une même chose. Le premier père fut traitre; la première mère menteuse; le premier fils voleur et fratricide. Qu'y a-t-il aujourd'hui qui n'ait existé jadis? Et qu'attendre de l'avenir? $»^{26}$

32 On retrouve dans la fiction picaresque le même péché originel qui a pour conséquence le même déterminisme qui pèse sur la vie du protagoniste. Guzmán, né dans une sorte de petit paradis terrestre (San Juan de Alfarache, d'où son nom) d'un père traître et d'une mère menteuse ne peut que devenir le délinquant sans honneur qui passe sa vie à commettre le péché. On voit bien ici le rôle d'exemplum que joue cette fiction picaresque dans le vaste sermon que représente l'oeuvre. Ce processus qui consiste à faire un sermon puis à l'illustrer avec la fiction romanesque est constant.

33 Ainsi, le thème du péché originel a pour corollaire le thème du libre arbitre, lui aussi omniprésent dans l'oeuvre. En effet, si du fait du péché originel l'homme est enclin à faire le mal, il peut en contrebalancer les effets par son libre arbitre qui lui permet chaque fois de choisir entre le bien et le mal. C'est tout le drame du gueux qui choisit invariablement de faire le mal, mais en toute connaissance de cause et il a la conscience très nette de prendre le chemin de l'enfer.

34 Enfin, les thèmes du péché originel et du libre arbitre débouchent sur le problème de la grâce qui à l'époque d'Alemán passionnait autant le grand public que les théologiens et qui suscitait des controverses passionnées dont on trouve un écho dans le Guzmán. Pour résumer disons que les dominicains, avec à leur tête Báñez, étaient les tenants de la prédestination de l'homme et affirmaient que Dieu accorde sa grâce comme il l'entend, même à des criminels, car ses raisons sont insondables. Leurs adversaires, les jésuites dont le porte-parole était Molina, insistaient au contraire sur l'importance des actions humaines car, selon eux, l'homme peut, par ses oeuvres, mériter la grâce divine. La polémique faisait rage au moment de la publication du Guzmán, et les dominicains, qui en avaient appelé à l'autorité du pape Paul V, étaient sur le point de l'emporter. ${ }^{27}$

Alemán semble hésiter entre les deux car, s'il affirme l'existence de la prédestination, il insiste aussi beaucoup sur l'efficacité des bonnes actions. Mais c'est tout de même la prédestination qui l'emporte car la fiction romanesque en est une illustration magistrale et on comprend alors le choix par Alemán d'un antihéros picaresque, choix qui rend sa démonstration plus éclatante et plus convaincante car si un criminel endurci comme Guzmán peut être illuminé par la grâce divine (le récit s'achève alors que Guzmán, sur la galère, après un véritable calvaire, est touché par la grâce et décide de choisir définitivement le bien), donc, si le plus misérable des hommes peut trouver son salut, à plus forte raison, chacun de nous peut être touché par la miséricorde divine. C'est la grande thèse du livre. Maurice Molho, qui qualifie le Guzmán de "géniale hyperbole de théologien » l'exprime magnifiquement en ces termes : « Le corps et le coeur dont Alemán a doté son gueux ne sont, à vrai dire, que la charpente charnelle d'une métaphysique. Guzmán d'Alfarache ne raconte pas l'histoire d'un homme, mais celle d'une âme, saisie dans son mouvement pendulaire. C'est une dialectique qui lui tient lieu de psychologie. $\mathrm{Ne}$ nous y trompons pas: Alemán ne semble s'être proposé d'autre objet dans son livre que l'analyse des lois de mécanique spirituelle qui régissent en toute âme l'affrontement du bien et du mal. $»^{28}$ 
36 Le Guzmán de Alfarache apparaît donc comme un vaste traité théologico-moral illustré par une infinité d'exempla parmi lesquels le plus important est la fiction picaresque. Et c'est sans doute en pensant à cette oeuvre que le critique espagnol, Miguel Herrero García a pu dire : «Le roman picaresque est un sermon avec une modification des proportions des éléments qui entrent dans sa composition. ${ }^{29}$ (c'est-à-dire la partie doctrinale et les exempla qui l'illustrent)

37 Le Guzmán de Alfarache, ce vaste sermon qui dénonce à saciété la nature mauvaise de l'homme et qui donne de la société et des relations humaines une vision noire, aboutit cependant à une conclusion inattendue. En effet, le gueux, forçat aux galères, après un supplice qui en a fait un déchet humain, connaît une nuit une illumination qui le métamorphose en ascète. Il se sent régénéré et il fait une vibrante profession de foi chrétienne. Il a définitivement choisi le Bien. Cette conversion inattendue laisse entrevoir, par-delà le désespoir de l'homme sur terre, un suprême espoir de salut pour le pécheur repenti. C'est finalement une conclusion optimiste qui transcende le profond pessimisme qui remplit le roman.

38 Enfin, en ce qui concerne le plan de l'expression, Alemán utilise une langue d'une abondance extraordinaire et une grande variété de styles. Dans les digressions idéologiques on trouve la prose de l'éloquence, faite de phrases longues, au tempo lent. C'est le style oratoire des sermons qui met à profit toutes les ressources de la réthorique (beaucoup de paragraphes, voire de chapitres commencent par une définition, suivie d'un dévelopement argumentatif illustré d'un exemplum ou de plusieurs exempla). Le contraste est grand entre cette prose d'idées et la prose vive, expressive des aventures picaresques émaillée de nombreuses expressions populaires, de proverbes, de jeux de mots, avec l'emploi d'un lexique d'une variété extrême, de dialogues pleins de vivacité. ${ }^{30}$

39 On voit, pour conclure, les grandes affinités qui rapprochent le Guzmán de Alfarache de la prose d'idées. Que ce soit au niveau thématique (satire sociale, satire morale), au niveau réthorique (structure du discours, argumentation) ou au niveau stylistique (prose de l'éloquence), une grande partie de l'oeuvre (celle que Lesage avait supprimée comme superflue, et que certains lecteurs ont sans doute tendance à sauter) peut indéniablement être qualifiée de prose d'idées. Ce qui l'en distingue, c'est, bien sûr, la présence, parmi les innombrables exempla, d'un immense exemplum fictionnel constitué par le récit autobiographique de la vie du gueux qui en fait un roman picaresque et non un simple traité théologico-moral. Les qualités de romancier de Mateo Alemán sont telles qu'elles font passer au second plan celles du penseur qui pourraient l'égaler aux meilleurs auteurs de prose d'idées de son époque. C'est pourquoi le Guzmán de Alfarache, unanimement considéré comme l'archétype du roman picaresque dont le Lazarillo de Tormes, génial lui aussi à sa manière, n'était qu'un prototype, une maquette en quelque sorte, est à mon avis un chef-d'oeuvre de la littérature d'idées.

40 Après le Guzmán, le roman picaresque reviendra à la formule du Lazarillo de Tormes, c'està-dire à la primauté de la fiction. Que ce soit au XVII ${ }^{\mathrm{e}}$ siècle en Espagne, avec le Buscón de Quevedo ou La Pícara Justina de López de Ubeda, ou en Allemagne le Simplex Simplicissimus de Grimmelshausen (1669, histoire d'un naïf qui connaît des aventures tragi-comiques extraordinaires pendant la guerre de trente ans), au XVIII ${ }^{e}$ siècle en Angleterre avec Moll Flanders de Daniel Defoe (histoire d'une prostituée, voleuse, qui finit par se régénérer), plus jamais on n'écrira de roman picaresque rempli de prose d'idées comme l'est le Guzmán de Alfarache qui reste unique en son genre. 


\section{NOTES}

1. Cf. Marie-Laure Acquier, Enjeux et ressorts de la prose d'idées dans l'Espagne du XVIIIe siècle. Le cas d'Antonio López de Vega (1586?-1656?), ci-dessus.

2. Stéphane Pujol, La prose d'idées, l'essai et le dialogue philosophique, Histoire de la France littéraire, Michel Prigent (dir.), T.2, Classicismes. XVIIe-XVIIIe siècle, Paris, PUF Quadrige, 2006, p.701-731.

3. Pedro Alfonso, Disciplina clericalis (XIe siècle)

4. Don Juan Manuel, Conde Lucanor, ed. José M. Blecua, CCA, Madrid, 1969.

5. Vincent Jouve, L'effet-personnage dans le roman, P.U.F., 1992, p.83-85.

6. Marie-Laure Acquier, art. cit., p.2.

7. Horace « Omne tulit punctum qui mixit utile dulci », Art poétique.

8. La vida de Lazarillo de Tormes y de sus fortunas y adversidades, anonyme, Anvers, 1554. Traduction de Maurice Molho sous le titre Lazare de Tormes, ses fortunes et ses adversités, in Romans picaresques espagnols, Editions Gallimard, Bibliothèque de la Pléiade, 1968.

9. Ibid.

10. Ibid., p. XVIII.

11. Étant donné le nombre réduit de gens qui savaient lire et écrire, la lecture publique était très répandue.

12. Le mot n'existe pas encore à l'époque avec le sens que nous lui donnons aujourd'hui. Les écrivains espagnols utilisaient les mots « historia » ou « poema ».

13. Il a publié une hagiographie La vida y milagros de San Antonio de Padua, un ouvrage de linguistique Ortografía castellana et un panégyrique sur son protecteur, l'archevêque de México Fray García Guerra intitulé Sucesos de Fray García Guerra. Voir Francisco Rico, Introduction a La novela picaresca española, Ed. Planeta, Barcelone, 1967, p.XCI-CI. Dorénavant, je citerai le texte espagnol du Guzmán d'après cette édition.

14. Primera parte de Guzmán de Alfarache, atalaya de la vida humana, Madrid, 1599.

15. "Mas, como el fin que llevo es fabricar un hombre perfecto, siempre que hallo piedras para el edificio, las voy amontonando » G.A., PII, L1, ch.7, p.557.

16. « No es todo de mi aljaba; mucho escogí de doctos varones y santos », G.A., "Al discreto lector », ibid., p.94.

17. «Declaración para el entendimiento de este libro»: «... se presupone que Guzmán de Alfarache, nuestro pícaro, habiendo sido muy buen estudiante, latino, retórico y griego, ... después dando la vuelta de Italia en España, pasó adelante con sus estudios, con ánimo de profesar el estado de religión; mas por volverse a los vicios los dejó, habiendo cursado algunos años en ellos. El mismo escribe su vida desde las galeras... Y no es impropiedad ni fuera de propósito si en esta primera escribiere alguna doctrina; que antes parece muy llegado a razón darla un hombre de claro entendimiento, ayudado de letras y castigado del tiempo, approvechándose del ocioso de la galera. » Ibid., p.96.

18. «Me sorprendo que haya habido quien llevara su paciencia a dar remate a semejante sarta de sermones enfadosos y pedestres de la más ramplona filosofía. » Miguel de Unamuno, Ramplonería, in Ensayos, Madrid, 1918.

19. «No miras ni reparas en las altas moralidades de tan divinos ingenios y sólo te contentas de lo que dijo el perro y respondió la zorra ", Francisco Rico, La novela picaresca española, op. cit., p.92. 
20. « ... no te rías de la conseja y se te pase el consejo; ... no los eches como barreduras al muladar del olvido... Recoje, junta esa tierra... y te aseguro hallarás algún oro que te enriquezca » Ibid., p.94.

21. "Larga digresión he hecho y enojosa, ya lo veo; mas no te maravilles, que la necesidad adonde acudimos era grande. » G.A., P.I, L.2, ch.4, p.271.

22. «Ya le oigo decir a quien me está leyendo que me arronje a un rincón, porque le cansa oírme. Tiene mil razones, que como verdaderamente son verdades las que trato, no son para entretenimiento, sino para el sentimiento; no para la chacota, sino, para con mucho estudio ser miradas y muy remediadas » G.A., P.II, L.3, ch.3, p.774.

23. «Porque con la purga no hagas asco y la dejes de tomar por el mal olor y sabor, echémosle un poco de oro, cubrámosla por encima con algo que bien parezca » G.A., P.II, L.3, ch.3, p.774.

24. Cristóbal Pérez de Herrera, Discursos del amparo de los legítimos pobres y reducción de los fingidos, 1598.

25. "Todos vivimos en asechanza los unos de los otros » G.A., P.I, L.2, ch.4, p.280.

26. «Este camino corre el mundo. No comienza de nuevo, que de atrás le viene al garbanzo el pico. No tiene medio ni remedio. Así lo hallamos, así lo dejaremos. No se espere mejor tiempo ni se piense que lo fue el pasado. Todo ha sido, es y será una misma cosa. El primero padre fue alevoso; la primera madre mentirosa; el primer hijo ladrón y fatricida.? Qué hay ahora que no hubo, o qué se espera de lo porvenir » G.A., P.I, L.III, chap.1, p.355.

27. Voir à ce sujet Francisco Rico, op. cit., p.CXXXIX-CXLI, n.49.

28. M. Molho, Romans picaresques espagnols, op. cit., Introduction p. LVII.

29. «La novela picaresca es un sermón con alteración de proporciones de los elementos que entran en su combinación » Miguel Herrero García, Nueva interpretación de la novela picaresca, in Revista de Filología Española, XXIV, Madrid 1937.

30. Voir F. Rico, op. cit., p.CXLVII-CLXXVII.

\section{RÉSUMÉS}

La prose d'idées et la littérature ne sont pas des catégories textuelles étanches car si la première utilise abondamment des exempla fictionnels pour illustrer ses démonstrations, la seconde contient toujours une dimension idéologique plus ou moins importante et plus ou moins explicite. Il s'agit donc d'une affaire de "dosage » entre la matière idéologique et la matière fictionnelle, dosage qui permet en principe de ranger les oeuvres dans l'une ou l'autre catégorie, ce qui n'est pas toujours évident car certaines oeuvres se situent à la frontière entre les deux. C'est le cas du roman picaresque espagnol, facétieux et burlesque, mais qui véhicule aussi dès les origines une indéniable satire sociale et morale. Le chef-d'œuvre du genre, le Guzmán de Alfarache de Mateo Alemán, se distingue par sa structure extrêmement digressive. Les aventures picaresques du protagoniste sont constamment entrecoupées de discours idéologiques sur toutes sortes de sujets touchant à la société et à la morale, discours entrelardés de cellules narratives secondaires qui font office d'exempla. L'ensemble de ces «sermons", d'un pessimisme profond, a sa source dans la doctrine religieuse catholique longuement exposée et appliquée rigoureusement et dans laquelle se détachent les thèmes fondamentaux du déterminisme, donc de la prédestination, et du libre arbitre de l'homme. Le Guzmán de Alfarache apparaît ainsi comme un vaste traité théologico-moral illustré par une infinité d'exempla parmi lesquels la fiction 
picaresque, l'histoire du gueux, est aussi un exemplum, le plus vaste et le plus important, au service de la thèse centrale du livre : la possibilité de rédemption de l'homme, fût-il le criminel le plus endurci, s'il est sauvé par la miséricorde divine. A tous les niveaux, thématique, réthorique et stylistique, le Guzmán de Alfarache a donc de grandes affinités avec la prose d'idées. Il s'en distingue par la proportion énorme de matière fictionnelle qu'il contient et par les qualités littéraires d'Alemán qui en font un roman et non un simple traité, ou, autrement dit, un chefd'œuvre de la littérature d'idées.

La prosa de ideas y la literatura no son categorías textuales estancas porque si la primera se vale con abundancia de exempla ficcionales para ilustrar sus demostraciones, la segunda contiene siempre una dimensión ideológica más o menos importante y más o menos explícita. Se trata pues de una cuestión de "dosificación » entre la materia ideológica y la materia ficcional, dosificación que permite en principio de clasificar las obras en una u otra categoría, lo que no resulta siempre evidente ya que algunas obras se sitúan en la frontera entre las dos. Este es el caso de la novela picaresca española, cómica y burlesca, pero que también encierra desde los orígenes una sátira social y moral innegable. La obra maestra del género, el Guzmán de Alfarache de Mateo Alemán, se distingue por su estructura digresiva en extremo. Las aventuras picarescas del protagonista están entrecortadas a cada paso por discursos ideológicos sobre toda suerte de asuntos relacionados con la sociedad y la moral, discursos salpicados de células narrativas secundarias que hacen las veces de exempla. El conjunto de dichos «sermones ", hondamente pesimistas, procede de la doctrina religiosa católica largamente expuesta y aplicada rigurosamente y en la cual se destacan los temas fundamentales del determinismo, pues de la determinación, y del libre albedrío del hombre. El Guzmán de Alfarache parece ser así un amplio tratado telológico-moral ilustrado por un sin fin de exempla entre los cuales la ficción picaresca, o sea la historia del pícaro, también es un exemplum, el más amplio y el más importante, al servicio de la tesis cental del libro : la posibilidad de redención del hombre, aunque fuera el criminal más empedernido, si le salva la misericordia divina. A todos los niveles, temático, retórico y estilístico, tiene pues el Guzmán de Alfarache afinidades grandes con la prosa de ideas. Se diferencia de ella por la proporción de materia ficccional que contiene y por las cualidades literarias de Alemán que hacen de él una novela y no un mero tratado, o, dicho de otra manera, una obra maestra de la literatura de ideas.

\section{INDEX}

Mots-clés : Espagne, littérature, prose d'idées, Siècle d'Or, roman picaresque

Index chronologique : Siècle d'Or, XVIe siècle, XVIIe siècle

\section{AUTEUR}

\section{JEAN-LOUIS BRAU}

Professeur émérite de l'Université de Nice Sophia-Antipolis, spécialiste de littérature espagnole du Siècle d'Or (XVI ${ }^{\mathrm{e}}$-XVII ${ }^{\mathrm{e}}$ siècles). Il est l'auteur d'articles sur le roman picaresque, le roman pastoral, l'œuvre de Cervantès et d'une thèse de Doctorat d'Etat sur la nouvelle intercalée dans le roman espagnol au Siècle d'Or (Cahiers de narratologie $\left.\mathrm{N}^{\circ} 4\right)$. 